\title{
Assessment of urinary microparticles in normotensive patients with type 1 diabetes
}

\author{
Yuliya Lytvyn $^{1}$ - Fengxia Xiao ${ }^{2}$ - Christopher R. J. Kennedy ${ }^{2} \cdot$ Bruce A. Perkins $^{3}$ • \\ Heather N. Reich ${ }^{4}$ - James W. Scholey ${ }^{4}$ David Z. Cherney ${ }^{4}$ • Dylan Burger ${ }^{2,5}$
}

Received: 25 September 2016 / Accepted: 30 November 2016/Published online: 21 December 2016

(C) Springer-Verlag Berlin Heidelberg 2016

\begin{abstract}
Aims/hypothesis Assessment of urinary extracellular vesicles including exosomes and microparticles (MPs) is an emerging approach for non-invasive detection of renal injury. We have previously reported that podocyte-derived MPs are increased in diabetic mice in advance of albuminuria. Here, we hypothesised that type 1 diabetes and acute hyperglycaemia would increase urinary podocyte MP levels in uncomplicated diabetes.

Methods In this post hoc exploratory analysis, we examined archived urine samples from normoalbuminuric patients with uncomplicated type 1 diabetes studied under clamped euglycaemia and hyperglycaemia and compared with healthy controls. Urinary vesicles were assessed by electron microscopy and nanoparticle tracking while podocyte MPs were assessed by flow cytometry.
\end{abstract}

David Z. Cherney and Dylan Burger contributed equally to this study.

Electronic supplementary material The online version of this article (doi:10.1007/s00125-016-4190-2) contains peer-reviewed but unedited supplementary material, which is available to authorised users.

Dylan Burger

dburger@uottawa.ca

1 Department of Pharmacology and Toxicology, University of Toronto, Toronto, ON, Canada

2 Kidney Research Centre, Ottawa Hospital Research Institute, 451 Smyth Rd, Ottawa, ON, Canada K1H 8M5

3 Department of Medicine, Division of Endocrinology, Mount Sinai Hospital, University of Toronto, Toronto, ON, Canada

4 Department of Medicine, Division of Nephrology, University Health Network, University of Toronto, Toronto, ON, Canada

5 Department of Cellular and Molecular Medicine, University of Ottawa, Ottawa, ON, Canada
Results Neither vesicle size nor total number were significantly altered in type 1 diabetes or acute hyperglycaemia. By contrast, urinary podocyte MP levels were higher in type 1 diabetes $(0.47$ [0.00-3.42] MPs/ $\mu \mathrm{mol}$ creatinine $[\mathrm{Cr}]) \mathrm{com}-$ pared with healthy controls $(0.00[0.00-0.00] \mathrm{MPs} / \mu \mathrm{mol} \mathrm{Cr}$, $p<0.05)$ and increased under hyperglycaemic clamp $(0.36$ [0.00-4.15] MPs/ $\mu \mathrm{mol} \mathrm{Cr}$ during euglycaemia vs 2.70 [0.00-15.91] MPs/ $\mu \mathrm{mol} \mathrm{Cr}$ during hyperglycaemia, $p<0.05)$. Levels of urinary albumin to creatinine ratio and nephrin (surrogates of podocyte injury) were unchanged by type 1 diabetes or acute hyperglycaemia.

Conclusion/interpretation Taken together, our data show that urinary podocyte MP levels are higher in patients with type 1 diabetes in advance of changes in other biomarkers (albuminuria, nephrin). Examination of podocyte MPs may serve as an early biomarker of glomerular injury in uncomplicated type 1 diabetes.

Keywords Albuminuria · Biomarker - Diabetes ·

Extracellular vesicle $\cdot$ Hyperglycaemia $\cdot$ Microparticle $\cdot$

Microvesicle $\cdot$ Podocyte

Abbreviations
$\begin{array}{ll}\text { DBP } & \text { Diastolic BP } \\ \text { miR } & \text { MicroRNA } \\ \text { MP } & \text { Microparticle } \\ \text { NTA } & \text { Nanoparticle tracking analysis } \\ \text { SBP } & \text { Systolic BP } \\ \text { TEM } & \text { Transmission electron microscopy }\end{array}$

\section{Introduction}

Extracellular vesicles are membrane-derived bilayered structures that are released by cells into the extracellular 
environment [1]. While nomenclature can be inconsistent, extracellular vesicles may be broadly sub-classified by size and mechanism of formation into exosomes, apoptotic bodies, and microparticles (MPs, also known as microvesicles) [1]. MPs $(0.1-1.0 \mu \mathrm{m})$ are characteristically formed through the outward blebbing of the plasma membrane following cell injury [1]. Because of the nature of their formation and the retention of phenotypic properties of the cells from which they originate, MPs are emerging biomarkers in vascular and renal disease $[1,2]$. In this regard, we recently reported that podocytederived MPs are increased in urine from diabetic mice in advance of albuminuria, suggesting that their enumeration may be useful in the identification of early podocyte injury in diabetes [2]. The purpose of the present study was to assess changes in urinary extracellular vesicles in patients with uncomplicated type 1 diabetes under clamped euglycaemia and hyperglycaemia compared with healthy controls under normoglycaemic conditions.

\section{Methods}

We conducted a post hoc exploratory analysis to assess urinary podocyte MPs in archived urine samples obtained from patients with uncomplicated type 1 diabetes compared with healthy controls. Healthy controls were studied during normoglycaemia and individuals with type 1 diabetes were studied under clamped euglycaemia (4-6 $\mathrm{mmol} / \mathrm{l})$ and hyperglycaemia (9-11 mmol/l). We performed a comprehensive characterisation of urinary vesicles using nanoparticle tracking analysis (NTA), transmission electron microscopy (TEM) and flow cytometry. Vesicle size and quantity were assessed and the utility of enumeration of urinary podocyte MPs was compared with existing surrogates of podocyte injury (nephrinuria, albuminuria). Studies were carried out in accordance with the Declaration of Helsinki, all study participants gave their informed consent and the study was approved by the University Health Network research ethics board.

For more detailed methods, please refer to the electronic supplementary material (ESM).

\section{Results}

Both healthy controls and participants with type 1 diabetes were young, normotensive and normoalbuminuric (Table 1). Baseline variables were similar between both groups, although heart rate was higher in the cohort with type 1 diabetes (Table 1). Study participants could not be taking antihypertensive or lipid-lowering agents. All patients were using an insulin pump or a multiple insulin dose regimen at the time of the screening visit. As expected, patients with type 1
Table 1 Baseline patient characteristics

\begin{tabular}{|c|c|c|}
\hline Variable & $\begin{array}{l}\text { Healthy controls } \\
(n=20)\end{array}$ & $\begin{array}{l}\text { Type } 1 \text { diabetes } \\
(n=25)\end{array}$ \\
\hline \multicolumn{3}{|l|}{ Baseline } \\
\hline Male & $10(50 \%)$ & $11(44 \%)$ \\
\hline Age (years) & $24.4 \pm 3.7$ & $25.0 \pm 5.2$ \\
\hline Diabetes duration (years) & - & $20.5 \pm 4.0$ \\
\hline BMI $\left(\mathrm{kg} / \mathrm{m}^{2}\right)$ & $23.3 \pm 2.8$ & $24.7 \pm 3.2$ \\
\hline $\mathrm{HbA}_{1 \mathrm{c}}(\%)$ & $5.3 \pm 0.3$ & $8.2 \pm 1.2^{*}$ \\
\hline \multicolumn{3}{|l|}{ Haemodynamic function } \\
\hline HR (bpm) & $64 \pm 8$ & $73 \pm 12^{*}$ \\
\hline $\mathrm{SBP}(\mathrm{mmHg})$ & $109 \pm 12$ & $106 \pm 20$ \\
\hline $\mathrm{DBP}(\mathrm{mmHg})$ & $64 \pm 7$ & $67 \pm 6$ \\
\hline GFR, $\mathrm{ml} \mathrm{min} \operatorname{mon}^{-1} 1.73 \mathrm{~m}^{-2}$ & $121 \pm 19$ & $144 \pm 35^{*}$ \\
\hline Urinary albumin/creatinine $(\mathrm{mg} / \mathrm{g})$ & $5.9 \pm 6.4$ & $5.7 \pm 5.5$ \\
\hline Serum creatinine $(\mu \mathrm{mol} / \mathrm{l})$ & $68.0 \pm 9.2$ & $58.0 \pm 8.1^{*}$ \\
\hline Podocyte MPs/ $/ \mu \mathrm{mol} \mathrm{Cr}$ & $0.00(0.00-0.00)$ & $0.47(0.00-3.42)^{*}$ \\
\hline
\end{tabular}

Values are mean \pm SD for normally distributed data or median and interquartile range for non-normally distributed data (podocyte MPs)

${ }^{*} p<0.05$ for healthy controls vs type 1 diabetic patients

$\mathrm{Cr}$, creatinine

diabetes exhibited higher GFR and $\mathrm{HbA}_{1 \mathrm{c}}$. Out of the 25 participants with type 1 diabetes, 14 exhibited normofiltration (56\%) and 11 hyperfiltration (44\%), where hyperfiltration was defined as GFR $\geq 135 \mathrm{ml} \mathrm{min}^{-1} 1.73 \mathrm{~m}^{-2}$.

Figure 1a shows the morphology of vesicles obtained following ultracentrifugation of pooled human urine. TEM revealed a heterogeneous mixture of structurally intact vesicles including exosomes (identified as $40-100 \mathrm{~nm}$ vesicles with cup-shaped morphology) and MPs (100-1000 nm vesicles with intact membrane). Rarely, apoptotic bodies (>1000 nm vesicles) were also seen. To further assess extracellular vesicle size and quantity, NTA was performed on cell-free urine. A size range from $\sim 30-1000 \mathrm{~nm}$ was observed by NTA. There were no differences in vesicle size or total vesicle concentration among the groups studied (not shown).

We subsequently assessed levels of podocyte-derived MPs in human healthy controls and type 1 diabetes samples using flow cytometry. Podocyte-derived MPs were identified by positive staining for both annexin $\mathrm{V}$ and podoplanin, with a size between $\sim 180 \mathrm{~nm}$ (lower limit of detection) and $\sim 1000 \mathrm{~nm}$. Using this approach the intra-assay variability was $6.6 \%$ and the inter-assay variability was $11.3 \%$. While the majority of healthy controls showed little to no detectable urinary podocyte MPs, levels of urinary podocyte MPs in type 1 diabetes were higher compared with healthy controls (Fig. 1b). In addition, levels of urinary podocyte MPs were increased in a subset of individuals during clamped hyperglycaemia compared with clamped euglycaemia (Fig. 1c). Nine participants had no detectable podocyte MP 
a

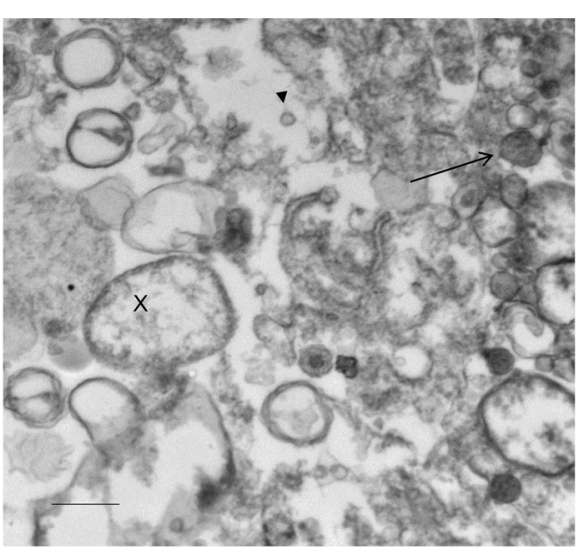

b

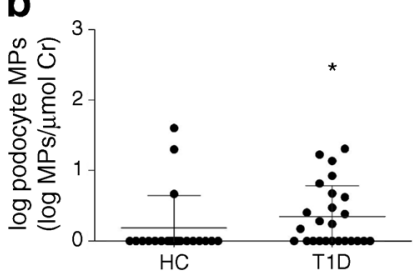

d

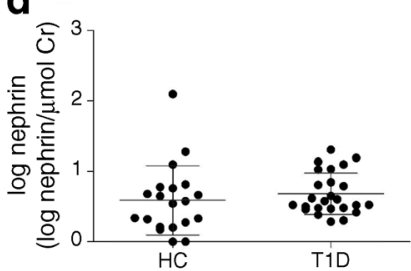

C

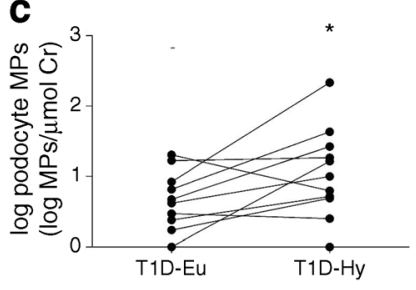

e

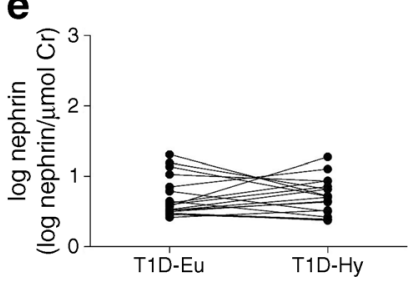

Fig. 1 MPs and nephrinuria in uncomplicated type 1 diabetes. (a) TEM image of the vesicle fraction of pooled urine samples; scale bar, $1 \mu \mathrm{m}$. The X symbol indicates apoptotic body, the arrow indicates MP and the arrowhead indicates exosome. (b) Levels of log-transformed podocyte MPs in urine from healthy controls (HC) $(n=20)$ and young adults with type 1 diabetes $(n=25)$. (c) Levels of log-transformed podocyte MPs in urine from participants with type 1 diabetes during clamped euglycaemia $(n=18)$ and hyperglycaemia $(n=18$; note overlap of 9 data points at 0 for T1D-Eu and overlap of 8 data points at 0 for T1D Hy). (d) Levels of logtransformed urinary nephrin from $\mathrm{HC}$ and young adults with type 1 diabetes. (e) Levels of log-transformed urinary nephrin from type 1 diabetes during clamped euglycaemia and hyperglycaemia. ${ }^{*} p<0.05$ vs HC, $n=18-25$. HC, healthy controls; T1D, participants with type 1 diabetes; T1D-Eu, T1D during clamped euglycaemia; T1D-Hy, T1D during clamped hyperglycaemia

excretion at euglycaemia. Of these individuals, 1/9 showed an increase in urinary podocyte MPs under hyperglycaemia while 8/9 showed no induction. There were no differences in podocyte MPs between patients with hyperfiltration and those with normofiltration $(p=0.375)$ and there were no correlations observed between podocyte MP levels and urinary albumin/ creatinine ratio $(p=0.18)$, inulin-clearance-based GFR $(p=0.90)$, systolic BP (SBP; $p=0.16)$, diastolic BP (DBP; $p=0.11)$, heart rate $(p=0.44)$ or diabetes duration $(p=0.45)$.

Nephrin release into the urine has also been proposed as a biomarker of podocyte injury in diabetes [3]. We therefore examined urinary nephrin levels by ELISA. As shown in

Fig. 1d, e, there were no differences in urinary nephrin levels between healthy controls and type 1 diabetes and no differences between clamped hyperglycaemia and clamped euglycaemia. Urinary nephrin levels showed no correlation with urinary podocyte MPs $(p=0.29)$.

\section{Discussion}

A growing body of evidence shows that cells ubiquitously release a diverse range of membrane vesicles of endosomal or plasma membrane origin [1]. Once released, these vesicles may be found in a host of biological fluids including plasma, cerebrospinal fluid, saliva, ascites fluid and urine [4]. Consistent with this, our TEM data of pooled urine samples revealed a heterogeneous mixture of exosomes, MPs and apoptotic bodies. While exosomes and MPs were commonly seen on micrographs, the occurrence of apoptotic bodies was rare.

Urinary extracellular vesicles may also reflect renal injury. For example, vesicles have been identified in the urine of patients with membranous nephropathy and focal segmental glomerular sclerosis by electron microscopy and aquaporin-2, neprisylin and podocalyxin were noted as vesicle-associated proteins [5]. More recently, alterations in subpopulations of urinary vesicles have been associated with structural changes in living donor kidneys [6]. With respect to diabetic nephropathy, microRNA (miR)-130a and miR-145 are increased and miR-155 and miR-424 are decreased in urinary exosomes from patients with microalbuminuria [7]. Levels of Wilm's tumour 1 are also altered in urinary exosomes in diabetic nephropathy and may be reflective of underlying injury [8]. Despite these advances, the majority of the above alterations were seen alongside overt renal injury (i.e. proteinuria or GFR decline). Urinary podocyte MPs are attractive surrogates of podocyte/glomerular injury for several reasons. First, MPs are formed under conditions of even mild cell stress thus offering direct insight into the earliest stages of injury. Second, podocyte MPs arise directly from podocytes which are among the earliest cellular targets in diabetes and are critical to the development of albuminuria. Third, quantification of MPs is achieved through a simple flow-cytometry-based approach without extensive proteomic or genomic testing. In the present study we measured levels of podocyte-derived MPs in urines of young adults with uncomplicated type 1 diabetes. Despite no evidence of renal injury, higher levels of urinary podocyte MPs were observed in this normo-albuminuric, normotensive cohort. These results support our previous work which found increased urinary podocyte MPs in diabetic mice and, to the best of our knowledge, represents the first evidence that levels of urinary podocyte MPs are higher in human diabetes.

This study has some limitations that should be considered when interpreting its findings. As this was the first examination of podocyte MPs in human diabetes we were unable to 
perform sample size calculations a priori. In addition, the impact of hyperglycaemia was only assessed in type 1 diabetes, not in healthy controls and only in a subset of individuals. Accordingly, it is unclear whether acute hyperglycaemia increases podocyte MP release under all conditions or if this effect is restricted to type 1 diabetes. Our previous work also showed that high glucose increases podocyte MP formation in vitro [2]. Consistent with this, podocyte MP levels were increased in type 1 diabetes under a $6 \mathrm{~h}$ hyperglycaemic clamp when compared with a euglycaemic clamp. In addition, as our study cohort consisted of individuals with type 1 diabetes without renal injury, the prognostic value of high urinary podocyte MPs is unclear at this time. It must be acknowledged that within the present study there was no relationship seen between podocyte MPs and measures of renal function (GFR). It is possible that the lack of a relationship simply reflects the early nature of disease in the samples analysed and that podocyte MP formation precedes increases in albuminuria and GFR decline; however, this remains to be tested. It is interesting to note that increases in podocyte MPs were seen in only approximately $30 \%$ of all samples analysed, as around one-third of all patients with diabetes will develop diabetic kidney disease [9]. Future studies should focus on the assessment of urinary podocyte MPs at all stages of diabetic kidney disease with the aim of establishing a temporal relationship between increases in urinary podocyte MP levels and the development of diabetic kidney disease. Such assessment between urinary podocyte MP levels and diabetic kidney disease should seek to determine whether higher levels are most associated with the development of albuminuria or with the initiation of decline in GFR.

In conclusion, this study has demonstrated for the first time that uncomplicated type 1 diabetes is associated with significantly higher urinary podocyte MP levels. Higher levels of podocyte MPs were seen in advance of other measures of glomerular injury (albuminuria, nephrinuria) and were independent of any changes in total urinary vesicles. Our studies set the stage for further, large-scale analysis of podocyte MPs as markers of early diabetic kidney injury.

Acknowledgements The authors acknowledge V. Tang from the University of Ottawa Flow Cytometry Core for assistance with flow cytometry analysis.

Data availability The data that support the findings of this study are available from the corresponding author upon reasonable request.
Funding DB is supported by grants from the Canadian Diabetes Association (OG-3-14-4548-DB), the Canadian Institutes of Health Research (362738), and a Kidney Research Scientist Core Education and National Training Program (KRESCENT) New Investigator Award. JWS is supported by grants from the Canadian Institutes of Health Research and the Heart and Stroke Foundation of Canada. YL was supported by a Canadian Diabetes Association Postdoctoral Fellowship and a Javenthey Soobiah Scholarship.

Contribution statement DB, YL and DZC designed and carried out the data analyses, interpreted the results and drafted the manuscript. FX contributed to the acquisition of data, designed the data analyses, interpreted the results and revised the manuscript critically for important intellectual content. CRJK contributed to analysis and interpretation of data and revised the manuscript critically for important intellectual content. BAP, HNR, and JWS contributed to the acquisition of data and revised the manuscript critically for important intellectual content. DB and DZC are the guarantors of this work and, as such, had full access to all the data in the study and take responsibility for the integrity of the data. All authors gave their final approval of this version of the manuscript.

Duality of interest The authors declare that there is no duality of interest associated with this manuscript.

\section{References}

1. Erdbrugger U, Le TH (2016) Extracellular vesicles in renal diseases: more than novel biomarkers? J Am Soc Nephrol 27:12-26

2. Burger D, Thibodeau JF, Holterman CE, Burns KD, Touyz RM, Kennedy CR (2014) Urinary podocyte microparticles identify prealbuminuric diabetic glomerular injury. J Am Soc Nephrol 25: 1401-1407

3. Camici M (2008) Urinary biomarkers of podocyte injury. Biomark Med 2:613-616

4. Gyorgy B, Szabo TG, Pasztoi M et al (2011) Membrane vesicles, current state-of-the-art: emerging role of extracellular vesicles. Cell Mol Life Sci 68:2667-2688

5. Rood IM, Deegens JK, Merchant ML et al (2010) Comparison of three methods for isolation of urinary microvesicles to identify biomarkers of nephrotic syndrome. Kidney Int 78:810-816

6. Turco AE, Lam W, Rule AD et al (2016) Specific renal parenchymal-derived urinary extracellular vesicles identify ageassociated structural changes in living donor kidneys. J Extracell Vesicles 5:29642

7. Barutta F, Tricarico M, Corbelli A et al (2013) Urinary exosomal microRNAs in incipient diabetic nephropathy. PLoS One 8:e73798

8. Kalani A, Mohan A, Godbole MM et al (2013) Wilm's tumor-1 protein levels in urinary exosomes from diabetic patients with or without proteinuria. PLoS One 8:e60177

9. Yee J (2008) Diabetic kidney disease: chronic kidney disease and diabetes. Diabetes Spectr 21:8-10 